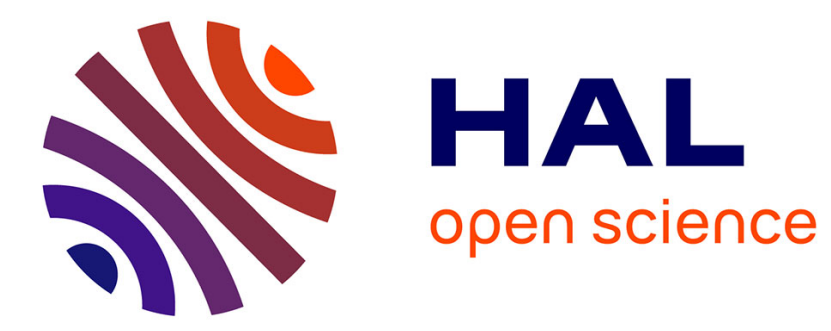

\title{
Système expert pour la conception en électronique de puissance
}

D. Fezzani, Hubert Piquet, H. Foch

\section{To cite this version:}

D. Fezzani, Hubert Piquet, H. Foch. Système expert pour la conception en électronique de puissance. Journal de Physique III, 1996, 6 (3), pp.349-361. 10.1051/jp3:1996100 jpa-00249461

\section{HAL Id: jpa-00249461 https://hal.science/jpa-00249461}

Submitted on 1 Jan 1996

HAL is a multi-disciplinary open access archive for the deposit and dissemination of scientific research documents, whether they are published or not. The documents may come from teaching and research institutions in France or abroad, or from public or private research centers.
L'archive ouverte pluridisciplinaire HAL, est destinée au dépôt et à la diffusion de documents scientifiques de niveau recherche, publiés ou non, émanant des établissements d'enseignement et de recherche français ou étrangers, des laboratoires publics ou privés. 


\title{
Système expert pour la conception en électronique de puissance
}

\author{
D. Fezzani, H. Piquet et H. Foch $\left(^{*}\right)$ \\ Laboratoire d'Électrotechnique et d'Électronique Industrielle $\left({ }^{* *}\right)$, I.N.P. Toulouse, \\ E.N.S.E.E.I.H.T., 2 Rue Charles Camichel, 31071 Toulouse cedex, France
}

(Reçu le 10 juillet 1995, accepté le 27 novembre 1995)

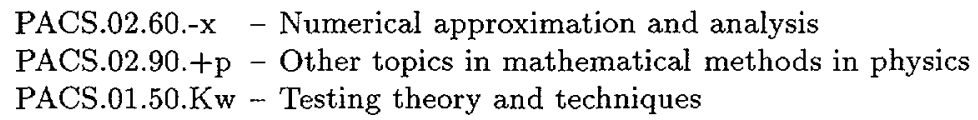

Résumé. - L'étude que nous proposons concerne la conception automatique des convertisseurs statiques et en particulier des Alimentations Sans Interruption (ASI). Le but recherché est la mise au point d'un outil apportant une aide à l'analyse du cahier des charges, au choix et au dimensionnement des structures les mieux adaptées. Pour réaliser la conception de ces ASI, une nouvelle approche est présentée. Les spécifications sont décrites par les performances recherchées au niveau des courants et des tensions à l'entrée et à la sortie. Ces données sont analysées par l'emploi de méthodes et de règles de conception permettant ainsi de dimensionner le filtre d'entrée, le filtre de sortıe, l'onduleur, le redresseur, etc. Au plan pratique, ces règles et méthodes sont implantées à l'aide du "générateur de système expert" SMECI. Nous présentons et justifions la structure modulaire qui a été mise en place, ainsi que son fonctionnement.

\begin{abstract}
The study that we propose concerns the automatic design of static converters and especially of Uninterrupted Power Supply (UPS). The aim is the development of a tool bringing an assistance in the analysis of the specifications, the choice and design of the structure that best match the application. To realize the design of these UPS, a new approach is presented. Specifications are described in terms of input and output performances (voltage, current, ...). These data are analyzed by using design rules and methods which allows designing the input filter, the output filter, the inverter, the rectifier, etc. In practice, these rules and methods are implemented on a micro computer by use of an "expert system generator" SMECI. We present and justify the modular structure that has been installed, as well as its functioning.
\end{abstract}

\section{Introduction}

L'étude présentée se situe dans le cadre de recherches dont l'objectif est la mise au point d'un système d'aide à la conception en électronique de puissance. Nous considérons ici le cas particulier des Alimentations Sans Interruption (ASI). En réponse aux contraintes d'un cahier des charges, il s'agit de déterminer la structure la mieux adaptée de l'onduleur d'une part, et du redresseur d'autre part, de définir le mode de commande et de contrôle de l'onduleur, de dimensionner les composants, d'évaluer les contraintes en courant et en tension qu'ils doivent supporter et enfin, de fournir les données nécessaires aux logiciels de simulation pour valider

$\left(^{*}\right)$ Auteur auquel doit ètre adressée la correspondance (Fax: (33) 61638875 )

$\left({ }^{* *}\right)$ U.R.A. au C.N.R.S. $n^{\circ} 847$

(C) Les Éditions de Physique 1996 


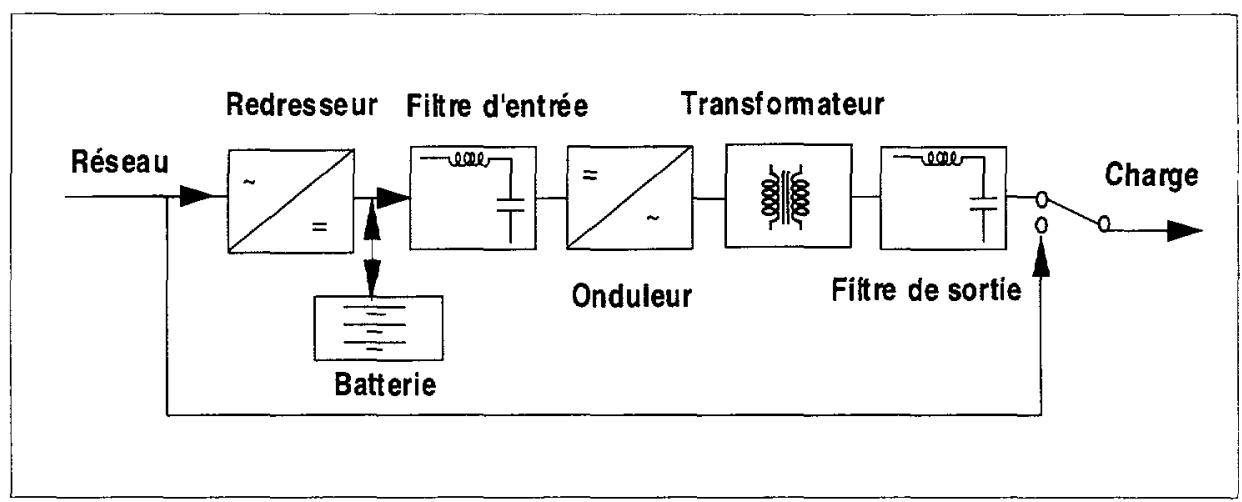

Fig. 1. - Schéma structurel d'une ASI.

[Structural diagram of an UPS.]

les choix proposés. Le savoir faire des concepteurs s'énonce généralement sous forme de règles ; aussi la mise en œuvre d'un "système expert» a-t-elle été envisagée.

Nous présentons ici, pour l'ensemble des modules développés, les résultats de cette approche et mettons en évidence l'intérêt de son application.

\section{Méthodes d'étude et de conception}

Dans le cas étudié ici (ASI) tout comme dans d'autres disciplines, la conception peut être considérée comme un ensemble de choix et de décisions permettant de déterminer un ensemble de paramètres qui constitue le but à satisfaire, en fonction de besoins exprimés à un instant donné, et définis par un cahier des charges.

L'analyse des spécifications permet en premier lieu le choix d'une structure appropriée pour chaque élément de cette ASI, dont la structure générale est présentée sur la figure 1.

En considérant une décomposition de l'ASI selon les éléments qui la constituent. il s'agit donc de définir et de caractériser : le redresseur, le filtre d'entrée, l'onduleur, le filtre de sortie et le transformateur.

Ces différents éléments interagissent au cours du fonctionnement et ne peuvent être définis de façon entièrement autonome.

Nous avons choisi de nous orienter vers un système qui reproduit la démarche de conception suivie par les «experts» car l'utilisation des moyens de simulation exigerait des balayages paramétriques lourds et longs.

Par ailleurs, nous souhaitons que ce système soit capable d'étendre et de compiler le «savoir faire» dans le domaine de la conception et par conséquent il doit être réutilisable, extensible et fiable. Autrement dit, il doit présenter un caractère modulaire.

Ceci nous a conduit à la réalisation de modules d'expertise autonomes. dédiés à la définition des différents éléments constitutifs de l'ASI (filtre d'entrée, redresseur, etc.). Ces modules ont une structure unique, modélisée dans un formalisme approprié pour faciliter l'intégration de nouvelles connaissances. 
Pour respecter cette modularité et prendre en compte l'interaction entre les différents modules, il a fallu établir un système leur permettant de coopérer.

Nous présentons maintenant le prototype de ce système implanté à l'aide du générateur de système expert SMECI [1].

\section{Définition des modules d'expertise}

Chacun des modules, associé aux éléments qui constituent l'ASI peut être caractérisé par :

- un but à déterminer ;

- un cahier des charges à satisfaire, où sont fixées les performances à atteindre ;

- des données supplémentaires pour le module qui concernent les conditions dans lesquelles il fonctionne ;

- des informations concernant le module où nous présentons tout ce qui, une fois le travail du module achevé, peut être utile pour apprécier les résultats fournis.

La fonction d'un module est donc, à partir de son cahier des charges, et ses données, de déterminer les valeurs présentes dans son but.

Il faut noter que cette représentation est unique pour tous les modules réalisés.

Notre description des modules de conception utilise la représentation à base de catégories (cette notion est équivalente à la notion de classes employée fréquemment dans les langages à objets) [1-4]. Cette représentation conduit à la description du problème à résoudre sous forme d'objets (instances des catégories décrites) : but à atteindre, cahier des charges, données et informations. Des champs sont définis au niveau de chaque catégorie pour représenter les propriétés des objets qui en sont issus. Les champs de l'objet sont, bien sûr, destinés à stocker des valeurs.

Il est possible d'associer aux catégories un ensemble de "méthodes» définissant le comportement des instances (les objets). Une méthode est une fonction (écrite en langage LE-LISP dans le cas de SMECI) rattachée au moyen de son nom à une catégorie [1].

La figure 2 présente à titre d'exemple les champs de la catégorie module filtre d'entrée : on y retrouve les 4 objets qui lui sont associés : son But, son Cahier, ses Infos et ses Données, ainsi que des connaissances procédurales, comprenant les méthodes (inductance 1 " et «condensateur 1 " qui permettent de calculer les valeurs $\left(L_{\mathrm{e}}\right.$ et $\left.C_{\mathrm{e}}\right)$ associées au But du filtre d'entrée, à partir des données et du cahier; ce dernier est défini par les ondulations de courant et de tension à ne pas dépasser $\left(\frac{\Delta I_{L}}{I_{L_{0}}}\right.$ et $\left.\frac{\Delta V_{C}}{V_{C_{0}}}\right)$ à l'entrée et à la sortie du filtre [5].

Les règles permettent de décrire le processus utilisé pour faire progresser la résolution du problème : elles manipulent les champs qui décrivent les objets et déclenchent les méthodes. La syntaxe utilisée pour les règles est quasiment celle du langage naturel. Par exemple, la règle choix-cellule- $L_{\mathrm{e}} C_{\mathrm{e}}$ présentée sur la figure 3 permet de dimensionner le filtre d'entrée de façon à atténuer les harmoniques de courant en sortie [5].

Cette règle fait apparaître deux parties principales :

- une première partie introduite par le mot clé Soit, permettant de déclarer des variables et sur lesquelles on applique des contraintes introduites par le mot clé Si ; 


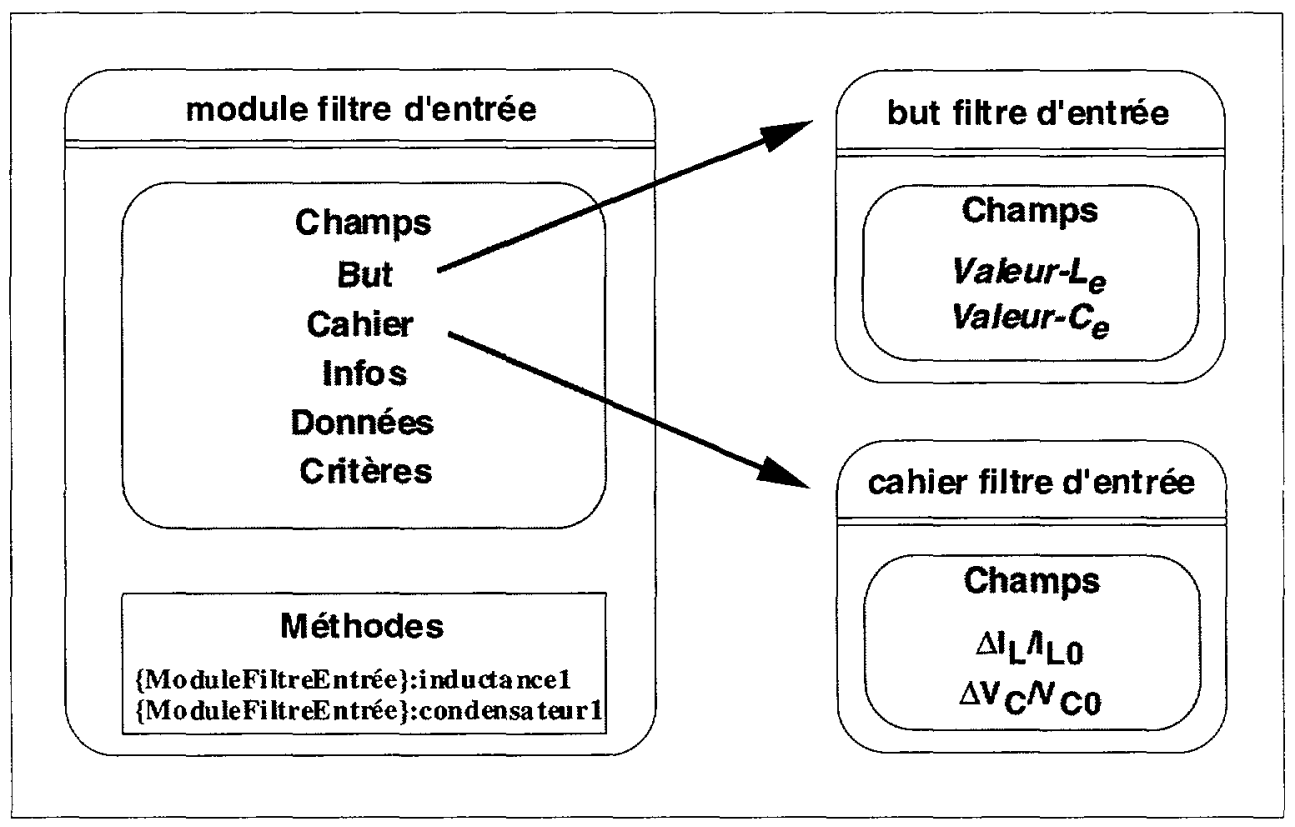

Fig. 2. - Champs des catégories associées au module filtre d'entrée.

[Slots of categories associated to the input filter module.]

\begin{tabular}{|c|c|c|}
\hline \multirow[t]{4}{*}{ Soit } & module un & module filtre d'entrée \\
\hline & Et cahıer & $=$ le Cahzer de module \\
\hline & Et but & $=$ le But de module \\
\hline & Et infos & $=$ les Infos de module \\
\hline \multirow[t]{3}{*}{$\mathrm{Si}$} & le Critères de module & $=$ minimisation-des-ondulations \\
\hline & Et le $\frac{\Delta I_{L}}{I_{L_{0}}}$ de cahzer & $<>0$ \\
\hline & Et le $\frac{\Delta V_{C}}{I_{C_{0}}}$ de cahrer & $<>0$ \\
\hline Alors & $\begin{array}{l}\text { la Valeur }-L_{e} \text { de but } \\
\text { la Valeur }-C_{e} \text { de but }\end{array}$ & $\begin{array}{l}=\$(\text { send 'inductance } 1 \text { module }), \text { et } \\
=\$(\text { send 'condensateur } 1 \text { module })\end{array}$ \\
\hline Action & & $\begin{array}{l}\$ \text { (print "J'ai calculé les valeurs de } L_{e} \text { et } \\
\text { de } C_{\mathrm{e}} \text { associées au but du fltre d'entrée.") }\end{array}$ \\
\hline
\end{tabular}

Fig. 3. - Exemple de règle introduite dans SMECI.

[Example of a rule as it has been implemented in SMECI.] 
Dans la règle ci-dessus, nous introduisons dans cette partie les variables module, cahier, but et infos qui dénotent respectivement des instances de la catégorie module flltre d'entrée et les objets qui lui sont associés. Dans la partie prémisse nous filtrons les objets retenus grâce aux contraintes : "si le critère de dimensionnement est la minimisation des ondulations ."

- une deuxième partie constituée de conclusions introduite par le mot clé Alors, présentant les conséquences logiques issues de la règle et d'actions introduites par le mot clé Action, permettant d'effectuer des actions externes (impression d'un message, . ).

Après avoir vérifié les trois contraintes, la règle choix-cellule- $L_{\mathrm{e}} C_{\mathrm{e}}$ procède à la détermination des valeurs des éléments du filtre en déclenchant les méthodes "inductance 1 " et "condensateur 1 ». Lorsque la règle est exécutée un message explicatif est affiché indiquant que le système a calculé les valeurs en questions.

Les règles écrites sont de type déductif, c'est à dire que si la partie introduite par Si, est vraie alors la partie introduite par Alors devient vraie. Ces règles sont organisées en bases de règles, elles-mêmes associées à des tâches. Cette organisation permet de structurer les connaissances et simplifie leur mise en œuvre.

Les modules filtre d'entrée, filtre de sortie et transformateur travaillent sur un circuit électrique bien défini dès le départ. Par contre, en ce qui concerne les modules onduleur et redresseur, une phase initiale consiste à déterminer la structure du convertisseur. Dans ce contexte, des méthodes de synthèse rigoureuses ont fait l'objet de plusieurs travaux $[6,7]$ déjà publiés et permettent, à partir de la caractérisation de la nature (source de courant ou de tension) des entrées et des sorties, de leur réversibilités (en courant et en tension), de procéder au choix de la structure.

\section{Présentation d'un module d'expertise}

Les modules d'expertise réalisés peuvent être considérés comme des systèmes autonomes dédiés à la Conception Assistée par Ordinateur permettant non seulement d'apporter une aide dans la conception de l'ASI, mais aussi de dimensionner le filtre d'entrée, le filtre de sortie, l'onduleur, .. de façon indépendante du reste de l'alimentation.

Chacune des connaissances identifiée (règles, "méthodes", catégories .. ) a été regroupée dans une base de connaissances. Ces dernières contiennent à la fois les informations relatives à l'application (la base de données du module) et toutes les connaissances nécessaires pour la conception.

Le LisP est utilisé comme langage de développement de ce logiciel, pour définir les «méthodes" des objets et des procédures dans les règles.

Dans la suite, nous allons présenter un module particulier que nous avons mis en place dans SMECI.

4.1. LE MODULE FILTRE D'ENTRÉE. - Le module filtre d'entrée a pour objectif de déterminer les paramètres d'un filtre qui répond aux exigences imposées par un cahier des charges en sélectionnant prioritairement certaines méthodes et critères mis au point [5]. Le cahier des charges est inspiré des conditions que doit remplir le filtre compte tenu de son rôle. Dans notre approche, le cahier des charges peut être défini par :

- l'ondulation résiduelle de la tension acceptable en sortie ;

- l'ondulation relative du courant fourni en entrée ; 


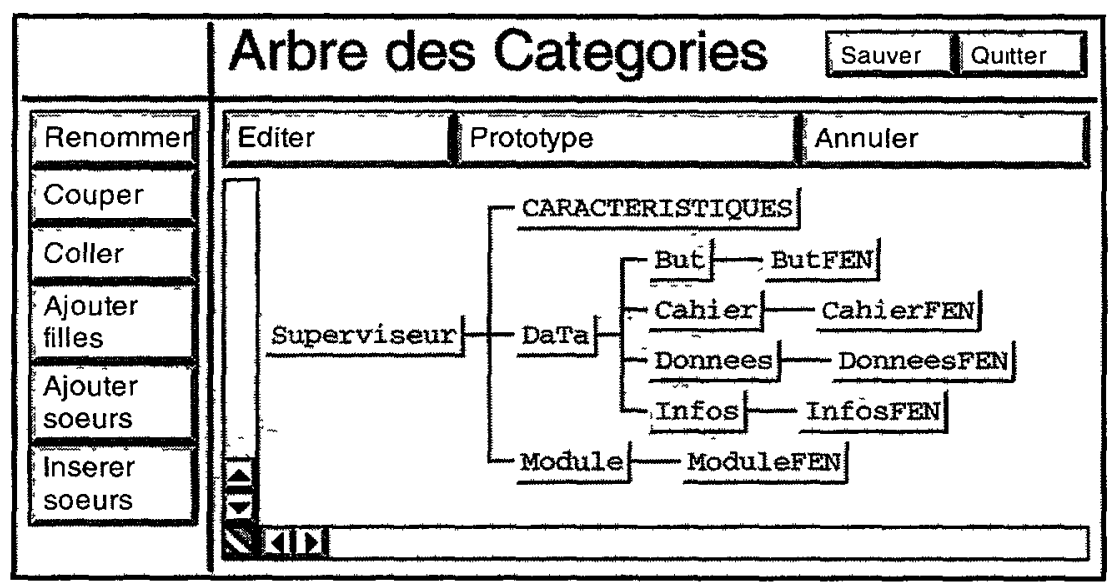

Fig. 4. - Décomposition du filtre d'entrée.

[Decomposition of the input filter.]

- le degré d'amortissement nécessaire ;

- la fréquence de coupure.

Les données de ce module peuvent être représentées par le spectre harmonique de la tension en entrée du filtre, le spectre harmonique du courant en sortie du filtre. la fréquence de la tension d'entrée, le facteur de qualité de l'inductance, etc.

Il reste à préciser qu'une fois le cahıer des charges et les données fournis, le travail du module consiste à déterminer son but c'est à dire l'obtention des valeurs de $L_{\mathrm{e}}$ et de $C_{\mathrm{e}}$.

Les informations caractérisent un certain nombre de résultats tels que les valeurs crêtes. efficaces des grandeurs électriques, les valeurs des résistances séries de l'inductance et du condensateur, etc.

Ces différentes catégories sont présentées sur l'arbre d'héritage de la figure 4.

La structure des objets est spécifiée par la description de leurs champs. La figure 5 présente les objets et les champs associés au module filtre d'entrée.

Le module filtre d'entrée a été configuré afin de reproduire au plus près la démarche schématisée par la figure 6 : la conception est décomposée en sous buts qui sont traités séquentiellement.

Le déclenchement de la règle de saisie des données permet à un utilisateur d'introduire les données ainsi que les spécifications du cahıer des charges ; l'analyse de ce dernier conduit à établir le modèle d'étude du filtre et à choisir le critère de dimensionnement à utiliser.

Si lors du "choix des spécifications》, le critère sélectionné est celui du choix de la fréquence de coupure du filtre (cf. Fig. 6) deux méthodes sont envisageables : la première consiste à associer la fréquence de coupure à l'ondulation relative du courant dans l'inductance à ne pas dépasser.

Une seconde possibilité consiste à utiliser une règle qui associe la fréquence de coupure à l'ondulation relative de la tension aux bornes du condensateur.

Un deuxième critère concernant la caractérisation en régime statique permet d'effectuer le dimensionnement du filtre, connaissant les ondulations relatives en entrée et en sortie.

Un dernier critère permet de caractériser le filtre en régime dynamique [5]. 

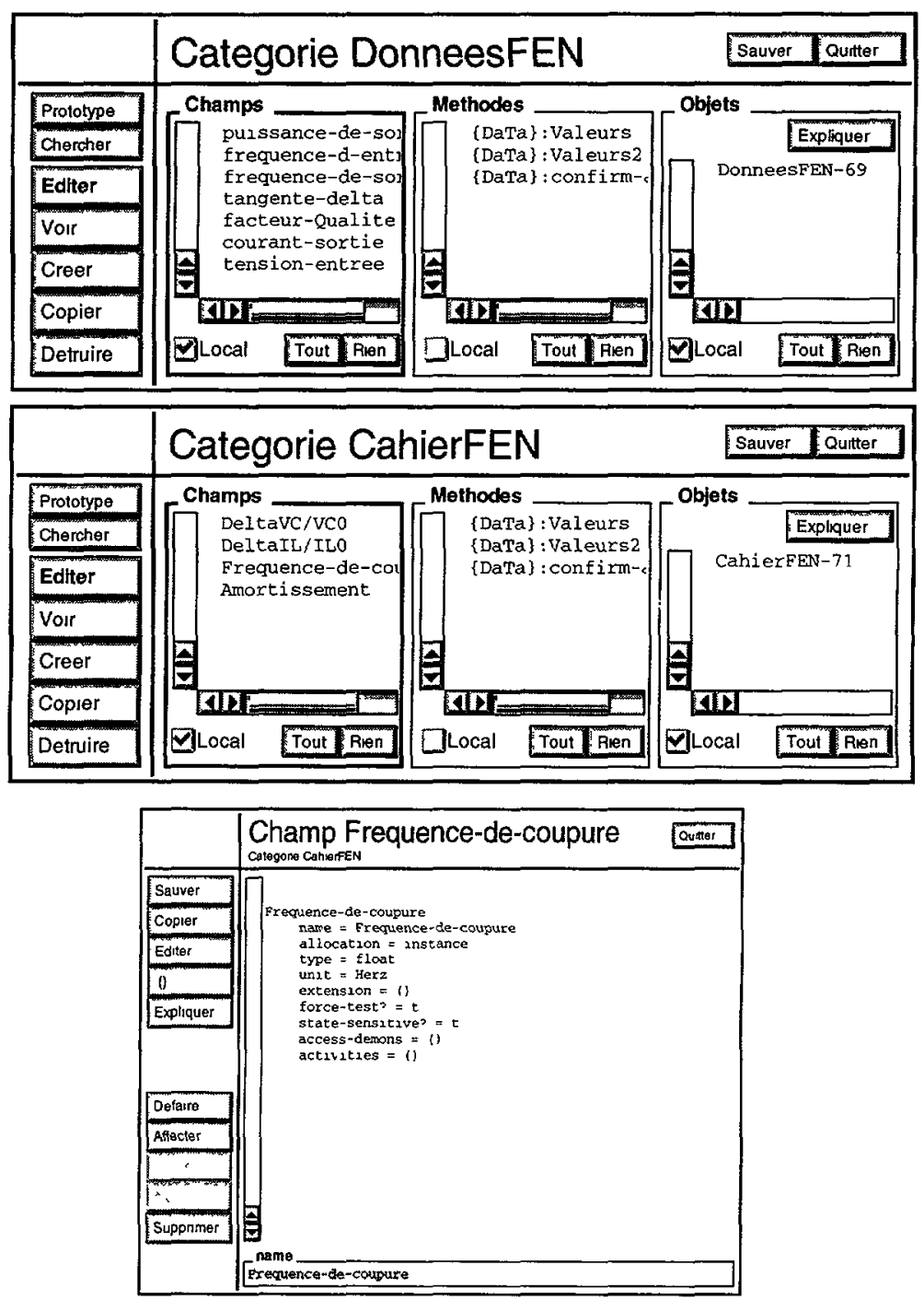

Fig. 5. - Catégories et champs associés au module filtre d'entrée.

[Classes and slots associated to the input filter module.]

Suivant les exigences de l'utilisateur, les règles s'enchaînent pour former différents chemins de déduction.

Cette méthode de conception, qui repose sur plusieurs critères ne donne pas une solution unique, et conduit à envisager un ensemble de solutions possibles. L'ajout de règles d'expert permettent de faire un choix parmi ces solutions proposées.

La conception peut se poursuivre par le calcul des valeurs crêtes du courant dans l'inductance et de la tension aux bornes du condensateur du filtre ainsi qu'un certain nombre d'informations supplémentaires. 
Choix des spécifications :

- amortissement

- fréquence de coupure

- ondulation $\Delta I_{L}$

- ondulation $\Delta V_{C}$

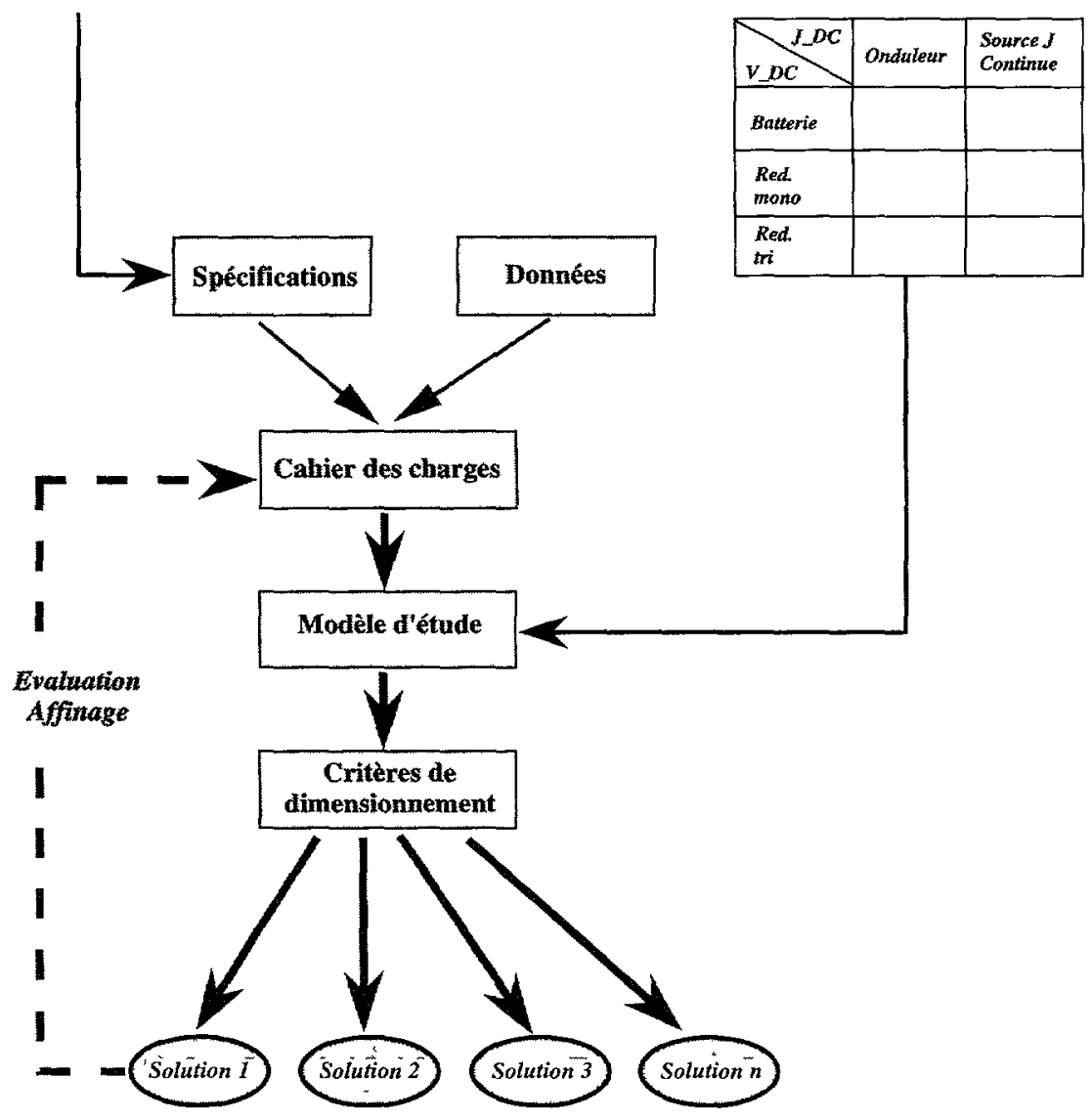

Choix des modèles d'étude:

- alimentation DC

- onduleur

\begin{tabular}{|l|l|l|}
\hline V_DC & Onduleur & $\begin{array}{l}\text { Sonrce J } \\
\text { Continue }\end{array}$ \\
\hline Baterie & & \\
\hline $\begin{array}{l}\text { Red. } \\
\text { mosto }\end{array}$ & & \\
\hline $\begin{array}{l}\text { Red. } \\
\text { tri }\end{array}$ & & \\
\hline
\end{tabular}

Fig. 6. - Modèle de raisonnement.

[Model of the design process]

Il reste à préciser que ce module fait appel à des programmes externes écrits en langage fortran' pour le calcul de la tension en sortie et du courant en entrée du filtre nécessaire pour la poursuite de la conception.

\section{Coopération entre les différents modules}

Liidée de base de cette représentation est la suivante : plusieurs "experts》 collaborent pour la conception et le dimensionnement de l'ASI, chacun apportant les compétences de son propre domaine et effectuant une tâche particulière. Le cahier des charges de l'ASI et les informations 


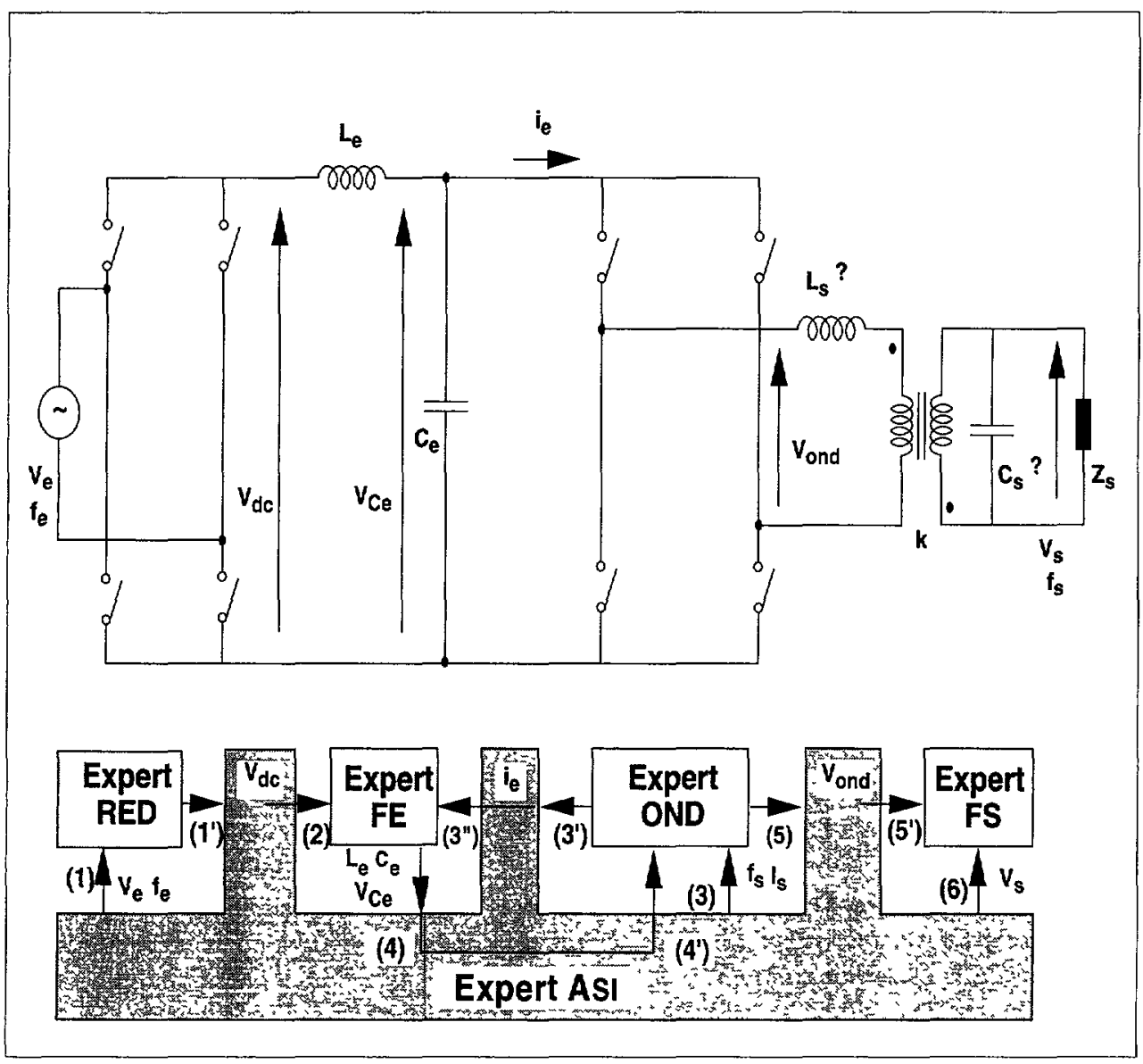

Fig. 7. - Modèle de coopération.

[Model of cooperation.]

fournies par l'utilisateur sont destinés à un module particulier : le module ASI chargé de piloter la conception.

Celui-ci fixe l'ordre dans lequel vont agir les différents "experts" (modules). En effet, comme les modules sont autonomes et ne communiquent pas directement entre eux, ils sont incapables de décider qui doit agir prioritairement. Le module ASI est le seul à posséder une vue d'ensemble du problème à résoudre. Il est chargé alors de choisir quel module doit agir à un instant donné en fonction de l'état de l'avancement de la conception. Il récupère les résultats obtenus par l'un des modules et les fournit aux autres pour faire évoluer la solution.

Pour illustrer ce propos, nous allons présenter une décomposition en étapes de la démarche de conception implantée sur "système expert" de l'ASI.

À chaque étape, on associe un ensemble de règles qui permettent d'atteindre un résultat intermédiaire dans la conception.

Nous présentons à titre d'exemple comment le système détermine les paramètres du filtre de sortie; le cahier des charges général et un certain nombre d'informations relatives à l'entrée 
et à la sortie sont fournies, au moyen de questions à l'utilisateur, au module ASI. Ce dernier analyse en premier lieu les données introduites et il en déduit que pour définir le filtre de sortıe, il faut déterminer la tension d'entrée de celui-ci. Cette tension dépend de celle fournie par le transformateur, elle-même dépendante de celle délivrée par l'onduleur, dépendante elle aussi des paramètres du filtre d'entrée.

Dans notre approche, le transformateur est considéré comme parfait, il est alors caractérisé par un simple rapport de transformation, sans dimension. Ainsi son rôle principal se réduit à l'adaptation des niveaux entre la tension délivrée par le filtre et la tension de sortie imposée par le cahier des charges. Le transformateur est inséré dans le filtre de sortze (Fig. 7) afin d'optimiser son fonctionnement.

L'interaction entre les modules conduit l'expert en ASI à procéder de la manière suivante, comme présentée sur la figure 7 :

- il fournit les informations concernant la tension $V_{\mathrm{e}}$ et la fréquence $f_{\mathrm{e}}$ en entrée au module redresseur (étape (1)) pour lui permettre de calculer la tension de sortie $V_{\mathrm{dc}}$ du redresseur (coopération entre le module ASI et le module redresseur),

- une fois la valeur de la tension $V_{\mathrm{dc}}$ connue, elle est récupérée par le module $A S I$ (coopération entre le module redresseur et le module ASI : étape (1')) qui se charge de la transmettre au module filtre d'entrée (étape (2)).

- il se trouve qu'à ce stade de la conception, une information nécessaire (courant de sortie $\imath_{\mathrm{e}}$ ) manque au module filtre d'entrée pour qu'il puisse déterminer les éléments de filtrage $\left(L_{\mathrm{e}}\right.$ et $\left.C_{\mathrm{e}}\right)$. Cette information est obtenue par l'intermédiaire du module ASI coopérant avec le module onduleur (étape (3)). L'onduleur détermine sa fonction de conversion et fournit le courant qu'il absorbe $i_{\mathrm{e}}$ au module $A S I$ (étape $\left(3^{\prime}\right)$ ), qui va une fois de plus faire appel au module filtre d'entrée (étape (3")) pour déterminer les valeurs de filtrage ainsi que la tension de sortie $V_{C_{r}}$,

- une fois la tension de sortie $V_{C .}$ du filtre d'entrée connue, elle est fournie (étape (4)) au module ASI (coopération entre le module filtre d'entrée et le module ASI). puis elle est transmise au module onduleur (coopération entre le module ASI et le module onduleur: étape $\left.\left(4^{\prime}\right)\right)$; celui-ci détermine sa tension de sortie $V_{\text {ond }}^{r}$.

- l'objectif suivant est enfin de déterminer les paramètres $\left(L_{\mathrm{s}}\right.$ et $\left.C_{\mathrm{s}}\right)$ du filtre de sortıe. À cette fin, le module ASI fait appel au module onduleur; celui-ci fournit sa tension de sortie $V_{\text {ond }}$ (étape (5)). Cette dernière est récupérée par le module $A S I$, puis transmise au module filtre de sortıe (étape $\left(5^{\prime}\right)$ ). Le module $A S I$ fournit également la valeur de la tension de sortie $V_{s}$, présente dans son cahier des charges au module filtre de sortie (étape (6)). Celui-ci possède alors les données nécessaires pour atteindre son but, c est à dire calculer les valeurs de $L_{\mathrm{s}}$ et $C_{\mathrm{s}}$.

Il faut noter que la conception peut se poursuivre :

- par la synthèse des interrupteurs de l'onduleur et du redresseur ;

- par l'évaluation des contraintes électriques des composants ;

- par la détermination des chronogrammes des commandes.

Elle peut être également affinée par l'adjonction et la caractérisation de l'environnement des interrupteurs : circuits d'aide à la commutation et évaluation des pertes. 


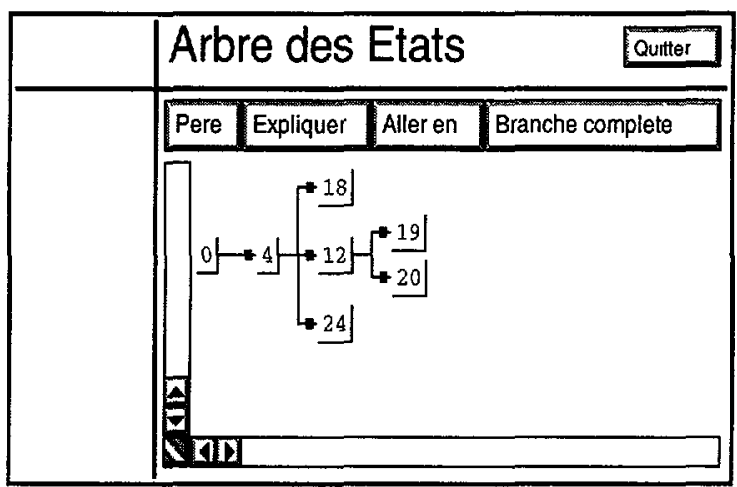

Fig. 8. - Arbre de résolution.

[Inference tree.]

\section{Fonctionnement du système}

Le système a été configuré afin de reproduire au plus près la démarche décrite au paragraphe 5 : la conception est décomposée en sous buts qui sont traités séquentiellement.

La réponse du système consiste en l'obtention des valeurs des composants, des contraintes électriques (valeurs crêtes, efficaces,. des courants et des tensions) et la commande des semi-conducteurs.

A u niveau de la mise en cuvre, le mode de déduction utilisé est le chaînage avant : le système part des faits connus et déclenche les règles dont les prémisses sont vérifiées, jusqu'à satisfaction du but courant.

Il se produit fréquemment au cours de la conception que plusieurs solutions soient envisageables. L'application des règles conduit à la création d'un arbre de résolution : dans celui-ci sont stockées toutes les modifications survenues lors de l'application des règles.

L'exploitation de cet arbre permet d'obtenir les différentes solutions atteintes et de fournir en langage naturel des explications et des démonstrations qui valident ses choix en exhibant les règles enchaînées au cours de la déduction.

En ce qui concerne le déroulement du raisonnement du système, la figure 8 présente l'arbre d'état créé par le système pendant la résolution, dans lequel on peut visualiser les différentes hypothèses appliquées, les solutions et les contradictions.

Tous les états ne sont pas représentés. Chaque branchement correspond à des raisonnements basés sur des critères différents. Par ailleurs, cet arbre montre qu'il est possible de générer des branchements pour effectuer un raisonnement en parallèle sur plusieurs états. D'où l'avantage d'obtenir plusieurs solutions pour un même problème.

Chaque changement d'état correspond au déclenchement d'une règle ; un module d'explication est capable d'exhiber les différentes règles et de parcourir l'arbre d'état pour présenter le raisonnement qui permet d'obtenir une valeur particulière d'un champ. La figure 9 explique une partie des déductions qui ont conduit à la détermination des valeurs de filtrage dans le cas où le critère est celui de la minimisation des taux d'ondulation (état 14). 


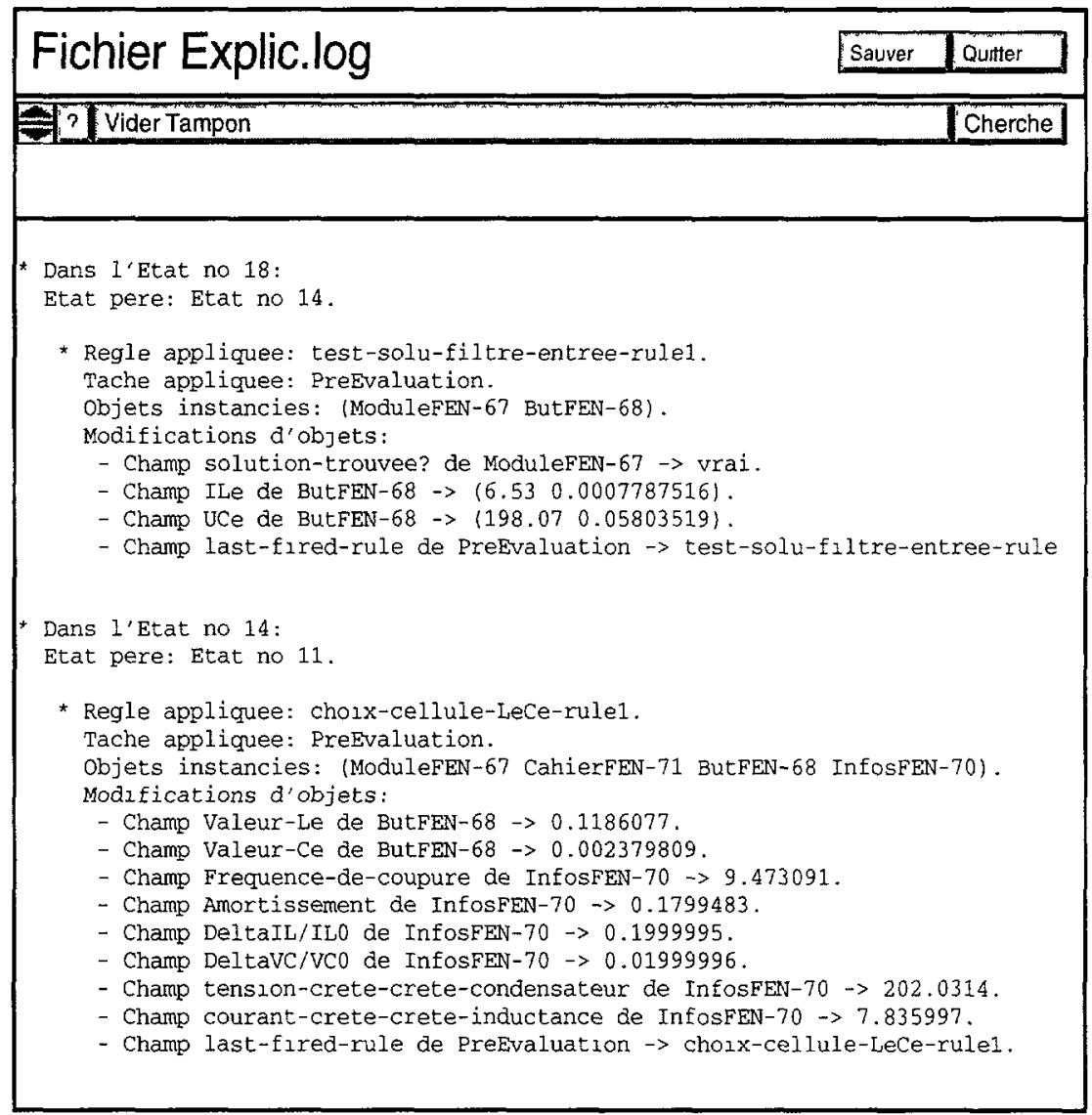

Fig. 9. - Explications sur un état.

[Explanations on a state.]

\section{Conclusion}

Nous proposons une approche de la conception en électronique de puissance basée sur l'utilisation des "systèmes à base de connaissances". La caractérisation des éléments constitutifs de l'ASI selon différents critères et données du cahier des charges a permis de mettre au point un prototype de conception pour les ASI. Celui-ci tente de reproduire la démarche diun expert humain et trouve son application dans le domaine de recherche.

Grâce à sa conception modulaire, les éléments mis en œuvre dans ce système sont réutilisables pour mettre au point de nouveaux outils de conception dédiés à d'autres structures de convertisseurs.

Comme outil de conception, il est capable de procéder à l'application automatique de règles générales à tout cas particulier spécifié par le cahier des charges.

Sur le plan pédagogique, il propose une démarche intellectuelle rigoureuse pour concevoir une structure et favorise l'apprentissage de cette méthode auprès des étudiants. 


\section{Bibliographie}

[1] SMECI, Manuel de référence, Version 1.65, (ILOG, 2 avenue Galliéni, BP 85, 94253 Gentilly, France 1992).

[2] Meyer B., Conception et programmation par objets pour du logiciel de qualité (InterEditions, Paris, 1990).

[3] Galloüin J.F., Transfert des connaissances ; systèmes experts : techniques et méthodes (Eyrolles, Ed., Paris, 1988).

[4] Laurière J., Intelligence artificielle : résólution de problèmes par l'homme et la machine (Eyrolles, Ed., Paris, 1987).

[5] Fezzani D., Piquet H. et Chéron Y., Critères de dimensionnement du filtre d'entrée dans les alimentations sans interruption, Proc. journées JTEA (Hammamet, Tunisie, 1994) pp. 178-185.

[6] Fezzani D., Piquet H. et Chéron Y., Design of static converters: an expert-system approach, Proc. of the conference EPE (Brighton, U.K., Sept. 1993).

[7] Piquet H., Cambronne J.P., Saadate S. et Glaize C., Méthodologie et outils pour la conception en électronique de puissance, Proc. of the conference EPF (Ens Cachan, France, 1994) pp. 25-30. 